\title{
On Factorization of Multivariate Polynomials over Algebraic Number and Function Fields
}

\author{
Seyed Mohammad Mahdi Javadi \\ School of Computing Science \\ Simon Fraser University \\ Burnaby, B.C. Canada. \\ sjavadi@cecm.sfu.ca.
}

\begin{abstract}
We present an efficient algorithm for factoring a multivariate polynomial $f \in L\left[x_{1}, \ldots, x_{v}\right]$ where $L$ is an algebraic function field with $k \geq 0$ parameters $t_{1}, \ldots, t_{k}$ and $r \geq 0$ field extensions. Our algorithm uses Hensel lifting and extends the EEZ algorithm of Wang which was designed for factorization over $\mathbb{Q}$. We also give a multivariate $p$-adic lifting algorithm which uses sparse interpolation. This enables us to avoid using poor bounds on the size of the integer coefficients in the factorization of $f$ when using Hensel lifting.

We have implemented our algorithm in Maple 13. We provide timings demonstrating the efficiency of our algorithm.
\end{abstract}

Categories and Subject Descriptors: I.1.2 [Symbolic and Algebraic Manipulation]: Algorithms - Algebraic algorithms;

General Terms: Algorithms, Theory.

Keywords: factorization algorithms, Hensel lifting, sparse interpolation, algebraic function fields.

\section{INTRODUCTION}

In a computer algebra system, computations with polynomials over algebraic function fields such as computing GCDs and factorization arise, for example, when one solves nonlinear polynomial equations involving parameters.

One way to factor $f \in L\left[x_{1}, \ldots, x_{v}\right]$ is to use Trager's algorithm [6]. His algorithm computes and factors the norm $(f)$ which is a polynomial in $x_{1}, \ldots, x_{v}$ over $\mathbb{Q}\left(t_{1}, \ldots, t_{k}\right)$. It exploits the fact that if $f_{i}$ is an irreducible factor of $f$ then firstly $h_{i}=\operatorname{norm}\left(f_{i}\right)$ is an irreducible factor of $\operatorname{norm}(f)$ and secondly $f_{i} \mid \operatorname{gcd}\left(f, h_{i}\right)$. One problem with this method is that the norm $(f)$ can be much larger than $f$. For example consider the following polynomial from Kotsireas [2].

$f=\frac{19}{2} c_{4}^{2}-\sqrt{11} \sqrt{5} \sqrt{2} c_{5} c_{4}-2 \sqrt{5} c_{1} c_{2}-6 \sqrt{2} c_{3} c_{4}+\frac{3}{2} c_{0}^{2}+\frac{23}{2} c_{5}^{2}+$

* Supported by NSERC of Canada and the MITACS NCE of Canada

Permission to make digital or hard copies of all or part of this work for personal or classroom use is granted without fee provided that copies are not made or distributed for profit or commercial advantage and that copies bear this notice and the full citation on the first page. To copy otherwise, to republish, to post on servers or to redistribute to lists, requires prior specific permission and/or a fee.

ISSAC'09, July 28-31, 2009, Seoul, Republic of Korea.

Copyright 2009 ACM 978-1-60558-609-0/09/07 ...\$5.00.

\author{
Michael Monagan \\ Department of Mathematics \\ Simon Fraser University \\ Burnaby, B.C. Canada. \\ mmonagan@cecm.sfu.ca.
}

$\frac{7}{2} c_{1}^{2}-\sqrt{7} \sqrt{3} \sqrt{2} c_{3} c_{2}+\frac{11}{2} c_{2}^{2}-\sqrt{3} \sqrt{2} c_{0} c_{1}+\frac{15}{2} c_{3}^{2}-\frac{10681741}{1985}$.

Here $L=\mathbb{Q}(\sqrt{2}, \sqrt{3}, \sqrt{5}, \sqrt{7}, \sqrt{11})$ is a number field and $f \in$ $L\left[c_{0}, \ldots, c_{5}\right]$. The norm of $f$ is degree 64 in $c_{0}, c_{1}, c_{2}, c_{3}, c_{4}, c_{5}$ and has about 3 million terms and the integers in the rational coefficients have over 200 digits so it is not easy to compute $\operatorname{norm}(f)$ let alone factor it. But we can easily discover that $f$ is irreducible by evaluating the variables $c_{0}, \ldots, c_{4}$ at small integers and then using Trager's algorithm to factor $\operatorname{norm}(f)$, a polynomial of degree 64 in $c_{5}$ over $\mathbb{Q}$. In this paper we generalize this to factor polynomials in $L\left[x_{1}, \ldots, x_{v}\right]$ using Hensel lifting. We evaluate all parameters and all variables except one at small integers thus reducing the factorization in $L\left[x_{1}, \ldots, x_{v}\right]$ to univariate factorization in $x_{1}$ over a number field. For notational purposes, we use $L(\alpha)$ to indicate the number field obtained by evaluating the parameters $t_{1}, \ldots, t_{k}$ in $L$ at an evaluation point $\alpha \in \mathbb{Z}^{k}$.

Some algorithms (See [7, 1, 10]) have been developed for factorization over an algebraic field $L$. A challenge is to solve the leading coefficient problem for lifting non-monic polynomials. Abbott in [1], suggests using a trick by Kaltofen in [4] which recursively computes the leading coefficients from their bivariate images using Hensel lifting. Our approach is to modify Wang's ingenious method given in [8] for factoring polynomials over $\mathbb{Z}$. His idea is to first factor the leading coefficient $l\left(x_{2}, \ldots, x_{v}\right)=\mathrm{lc}_{x_{1}}(f)$ of the input polynomial $f$ in the main variable $x_{1}$, recursively. Then evaluate all the variables except $x_{1}$ at an evaluation point $\alpha \in \mathbb{Z}^{v-1}$ and factor the univariate polynomial $f(\alpha)$. Now using the integer leading coefficients of the univariate factors, one can determine which factor of $l\left(x_{2}, \ldots, x_{v}\right)$ belongs to the leading coefficient of which factor of $f(\alpha)$. To do this, Wang identifies unique prime divisors for each factor of $l\left(x_{2}, \ldots, x_{v}\right)$ evaluated at $\alpha$ by computing integer GCDs only. Unfortunately this idea does not generalize to $L$. We show an example.

$$
\begin{aligned}
& \text { Example } 1 \text { Let } L=\mathbb{Q}(\sqrt{-5}) \text { and } \\
& \qquad \begin{aligned}
f & =((y+\sqrt{-5}+1) x+1)((y+\sqrt{-5}-1) x+1) \\
& =\left(y^{2}+2 \sqrt{-5} y-6\right) x^{2}+2(y+\sqrt{-5}) x+1
\end{aligned}
\end{aligned}
$$

We have $l c_{x}(f)=y^{2}+2 \sqrt{-5} y-6 \in L[y]$, so if we evaluate $y$ at $\alpha \in \mathbb{Z}$, we will obtain an element of $\mathbb{Z}[\sqrt{-5}]$. But $\mathbb{Z}[\sqrt{-5}]$ is not a unique factorization domain and $G C D$ s do not always exist in this ring. For example, for $y=0$ we have $l c_{x}(f)(y=0)=-6=-2 \times 3=-(1-\sqrt{-5}) \times(1+\sqrt{-5})$.

Another problem is that one needs to do computations with fractions in Hensel lifting. To solve this problem, one 
can work modulo a power of a prime, $p^{l}$. This modulus, $p^{l}$, must be at least twice the largest integer coefficient in any factor of $f$. Unfortunately the known bounds on the sizes of the integer coefficients in the factors of $f$ are usually very big which makes the computations really slow. In [1] it is suggested that it is better not to do the calculations modulo $p^{l}$ because of the bad bounds but instead to lift over $\mathbb{Q}$. In our algorithm we choose a prime $p$ of a modest size and then lift the integer coefficients to their correct values using a new multivariate $p$-adic lifting algorithm which uses a sparse interpolation method similar to Zippel's algorithm [11].

Our paper is organized as follows. In Section 2 we present an example showing the main flow and the key features of our algorithm. We then identify possible problems that can occur and how the new algorithm deals with them in Section 3. In Section 4 we present our new algorithm. Finally, in Section 5 we compare Maple implementations of our algorithm with Trager's algorithm for a set of polynomials.

\section{AN EXAMPLE}

Let $F=\mathbb{Q}\left(t_{1}, \ldots, t_{k}\right), k \geq 0$. For $i, 1 \leq i \leq r$, let $m_{i}\left(z_{1}, \ldots, z_{i}\right) \in F\left[z_{1}, \ldots, z_{i}\right]$ be monic and irreducible over $F\left[z_{1}, \ldots, z_{i-1}\right] /\left\langle m_{1}, \ldots, m_{i-1}\right\rangle$. Let $L=F\left[z_{1}, \ldots, z_{r}\right] /$ $\left\langle m_{1}, \ldots, m_{r}\right\rangle . L$ is an algebraic function field in $k$ parameters $t_{1}, \ldots, t_{k}$ (this also includes number fields). Let $f$ be a non-zero square-free polynomial in $L\left[x_{1}, \ldots, x_{v}\right]$. Our problem is given $f$, compute $f_{1}, f_{2}, \ldots, f_{n}$ such that $f=$ $\mathrm{lc}_{x_{1}, \ldots, x_{v}}(f) \times f_{1} \times f_{2} \times \cdots \times f_{n}$ where $f_{i}$ is a monic irreducible polynomial in $L\left[x_{1}, \ldots, x_{v}\right]$.

Our algorithm works with the monic associate $\tilde{f}$ of the input $f$ and primitive associates of the minimal polynomials which we now define.

Definition 1 Let $D=\mathbb{Z}\left[t_{1}, \ldots, t_{k}\right]$. A non-zero polynomial in $D\left[z_{1}, \ldots, z_{r}, x_{1}, \ldots, x_{v}\right]$ is said to be primitive wrt $\left(z_{1}, \ldots, z_{r}, x_{1}, \ldots, x_{v}\right)$ if the $G C D$ of its coefficients in $D$ is 1 . Let $f$ be non-zero in $L\left[x_{1}, \ldots, x_{v}\right]$. The denominator of $f$ is the polynomial den $(f) \in D$ of least total degree in $\left(t_{1}, \ldots, t_{k}\right)$ and with smallest integer content such that den $(f) f$ is in $D\left[z_{1}, \ldots, z_{r}, x_{1}, \ldots, x_{v}\right]$. The primitive associate prim $(f)$ of $f$ is the associate of $\operatorname{den}(f) f$ which is primitive in $D\left[z_{1}, \ldots, z_{r}, x\right]$ and has positive leading coefficient in a term ordering. The monic associate $\tilde{f}$ of $f$ is defined as $\tilde{f}=\operatorname{prim}(\operatorname{monic}(f))$. Here monic $(f)$ is defined by $l c_{x_{1}, \ldots, x_{v}}(f)^{-1} f$.

Example 2 Let $f=3 t x^{2}+6 t x /\left(t^{2}-1\right)+30 t z /(1-t)$ where $m_{1}(z)=z^{2}-t$. Here $f \in L[x]$ where $L=\mathbb{Q}(t)[z] /\left\langle z^{2}-t\right\rangle$ is an algebraic function field in one parameter $t$. We have $\operatorname{den}(f)=t^{2}-1$ and $\tilde{f}=\operatorname{prim}(f)=\operatorname{den}(f) f /(3 t)=\left(t^{2}-\right.$ 1) $x+2 x-10 z(t+1)$. For $f=2 z x^{2}+2 / t$ we have $\operatorname{prim}(f)=$ $t z x^{2}+1, \operatorname{monic}(f)=x^{2}+z / t^{2}$ and $\tilde{f}=t^{2} x^{2}+z$.

We demonstrate our algorithm using the following example using $t$ for a parameter and $x$ and $y$ for variables.

Example 3 Let $m(z)=z^{2}-t^{3}+t$ and

$$
\begin{gathered}
f=\left(t^{3}-t\right) y^{2} x^{2}+\left(20 t^{3} z-t^{2} z-20 t z+z\right) y x^{2}+ \\
\left(-20 t^{5}+40 t^{3}-20 t\right) x^{2}+(-t z+21 z) y x+\left(421 t^{3}-421 t\right) x-21 t \\
=\left(t^{3}-t\right)\left(x y+20 z x-\frac{z}{t^{2}-1}\right)\left(x y-\frac{z x}{t}+\frac{21 z}{t^{3}-t}\right) .
\end{gathered}
$$

Here $L=\mathbb{Q}(t)[z] /\left\langle z^{2}-t^{3}+t\right\rangle$ and $f \in L[x, y]$. We have $\operatorname{prim}(f)=f$ and $\tilde{m}=m$. The first step in our algorithm is to eliminate any algebraic elements in $\gamma=l c_{x, y}(\operatorname{prim}(f))=$ $t^{3}-t$ by computing $\tilde{f}$. This is done to avoid any fractions in the parameter $t$ in the Hensel lifting. Since $\gamma$ does not involve the algebraic element $z$, we have $\tilde{f}=\operatorname{prim}(f)$.

Suppose we choose $x$ as the main variable. In order to use Hensel lifting we factor the leading coefficient

$l c_{x}(\tilde{f})=\left(t^{3}-t\right) y^{2}+\left(20 t^{3} z-t^{2} z-20 t z+z\right) y-20 t^{5}+40 t^{3}-20 t$.

We do this by recursively using our algorithm in one less variable. We will obtain

$$
l c_{x}(\tilde{f})=\gamma \times l_{1} \times l_{2}=\left(t^{3}-t\right)(y-z / t)(y+20 z) .
$$

Now we clear the denominators in $l_{i} s$ to obtain $l c_{x}(\tilde{f})=$ $\bar{\gamma} \times \tilde{l}_{1} \times \tilde{l}_{2}=\left(t^{2}-1\right)(t y-z)(y+20 z)$. In order to factor $\tilde{f}$, we evaluate it at a point $\alpha$ for all the parameters and variables except the main variable, $x$. We factor the resulting univariate polynomial in $\mathbb{Q}[z][x] /\langle\tilde{m}(\alpha)\rangle$ using Trager's algorithm and then we lift the variables and parameters one by one using Hensel lifting. Suppose we choose the evaluation point to be $\alpha=(t=12, y=5)$. This evaluation point must satisfy certain conditions that we will discuss in Section 3.2. We have

$$
\tilde{f}(\alpha)=(170885 z-4864860) x^{2}+(45 z+722436) x-252
$$

and $\tilde{m}(\alpha)=z^{2}-1716$ which is irreducible hence $L(\alpha)$ is a field. Using Trager's algorithm, we factor $\tilde{f}(\alpha)$ over $L(\alpha)$ to get

$$
\begin{gathered}
\tilde{f}(\alpha)=l c_{x}(\tilde{f}(\alpha)) \times u_{1} \times u_{2}=(170885 z-4864860) \times \\
\left(x+\frac{1}{19630325} z-\frac{48}{137275}\right) \times\left(x+\frac{105}{22451} z+\frac{21}{157}\right) .
\end{gathered}
$$

We factor $\bar{\gamma} \in \mathbb{Z}[t]$ to obtain $\bar{\gamma}=t^{2}-1=\tilde{l}_{3} \times \tilde{l}_{4}=(t-$ $1)(t+1)$. Before doing Hensel lifting, we determine the true leading coefficient of each factor of $\tilde{f}$. To do this, we use the denominators of $u_{1}$ and $u_{2}$. We know that

$$
d_{i}=\operatorname{den}\left(u_{i}\right) \mid \operatorname{den}\left(\frac{1}{l c_{x}\left(\tilde{f}_{i}(\alpha)\right)}\right)
$$

where $\tilde{f}_{i}$ is a factor of $\tilde{f}$. We have

$$
\begin{aligned}
d_{1} & =\operatorname{den}\left(u_{1}\right)=19630325=(5)^{2}(11)(13)(17)^{2}(19), \\
d_{2} & =\operatorname{den}\left(u_{2}\right)=22451=(11)(13)(157) \\
D_{1} & =\operatorname{den}\left(1 / \tilde{l}_{1}(\alpha)\right)=1884=(2)^{3}(3)(157) \\
D_{2} & =\operatorname{den}\left(1 / \tilde{l}_{2}(\alpha)\right)=(5)^{2}(17)^{2}(19) \\
D_{3} & =\operatorname{den}\left(1 / \tilde{l}_{3}(\alpha)\right)=11 \\
D_{4} & =\operatorname{den}\left(1 / \tilde{l}_{4}(\alpha)\right)=13 .
\end{aligned}
$$

The evaluation point $\alpha$ was chosen so that $D_{i}$ 's have a set of distinct prime divisors, namely $\{3,17,11,13\}$. Here $D_{i}$ 's are relatively prime so we have

$$
\operatorname{gcd}\left(d_{i}, D_{j}\right)>1 \Rightarrow \tilde{l}_{j} \mid \bar{l}_{i}
$$

where $\bar{l}_{i}=l c_{x_{1}}\left(\tilde{f}_{i}\right)$. Using this we obtain $\bar{l}_{1}=\left(t^{2}-1\right)(y+$ $20 z)$ and $\bar{l}_{2}=\left(t^{2}-1\right)(t y-z)$ and we have

$$
\tilde{f} \equiv \frac{1}{t^{2}-1} \times\left(\bar{l}_{1}(\alpha) u_{1}\right) \times\left(\bar{l}_{2}(\alpha) u_{2}\right) \bmod \langle t-12, y-5\rangle .
$$


To avoid fractions in $\mathbb{Q}(t)$ in the Hensel lifting we multiply

$$
\tilde{f}:=\frac{\bar{l}_{1} \times \bar{l}_{2}}{l c_{x_{1}}(\tilde{f})} \times \tilde{f}=\left(t^{2}-1\right) \times \tilde{f} .
$$

Now we use Hensel lifting to lift the parameter $t$ and the variable $y$ in the other coefficients of the $\tilde{f}_{i}$. To avoid any computations with fractions in $\mathbb{Q}$, we do the calculations modulo a prime, say $p=17$. After applying Hensel lifting we obtain the factors $\bar{f}_{1}=\left(\left(t^{2}-1\right)(y+20 z) x-z\right)$ and $\bar{f}_{2}=\left(\left(t^{2}-1\right)(t y-z) x+4 z\right)$ s.t. $\tilde{f} \equiv \bar{f}_{1} \times \bar{f}_{2}(\bmod 17)$. The final task is to find the integer coefficients of $\bar{f}_{1}$ and $\bar{f}_{2}$. To do this, we use sparse interpolation. We have $e_{1}=$ $\tilde{f}-\bar{f}_{1} \times \bar{f}_{2} \bmod \langle\tilde{m}\rangle$, the first error polynomial over $\mathbb{Z}$. We want to find $\sigma_{1}, \sigma_{2} \in L[x, y]$ s.t.

$$
\tilde{f} \equiv\left(\bar{f}_{1}+\sigma_{1} \times p\right)\left(\bar{f}_{2}+\sigma_{2} \times p\right) \bmod p^{2} .
$$

Assuming that our choice of $\alpha$ and $p$ has not caused any terms in the polynomials $\bar{f}_{1}$ and $\bar{f}_{2}$ to vanish, we know that the monomials in $\sigma_{1}$ and $\sigma_{2}$ are the same as those in $\bar{f}_{1}$ and $\bar{f}_{2}$ respectively, so we have the assumed forms for $\sigma_{1}$ and $\sigma_{2}$. Since $\bar{f}_{1}$ and $\bar{f}_{2}$ have correct leading coefficients we have $\sigma_{1}=A z$ and $\sigma_{2}=B z$ for unknown coefficients $A$ and $B$. To find the values for $A$ and $B$ we have

$$
\left(\sigma_{1} \times \bar{f}_{2}+\sigma_{2} \times \bar{f}_{1}\right) \bmod \langle\tilde{m}\rangle-\frac{e_{1}}{p} \equiv 0 \bmod p .
$$

After equating every coefficient in $x, y, z$ and $t$ in the above expression to zero, we get the following linear system:

$$
\begin{gathered}
\{A=0,-B+1=0, B-1=0,-1-4 A+B=0,1-B+4=0, \\
A=0,-A-20+20 B=0,2 A+40-40 B=0,-A=0\} .
\end{gathered}
$$

Solving modulo $p$, we get $A=0$ and $B=1$ so we update

$$
\bar{f}_{1}:=\bar{f}_{1}+\sigma_{1} \times p=\left(\left(t^{2}-1\right)(y+20 z) x-z\right)
$$

and

$$
\bar{f}_{2}:=\bar{f}_{2}+\sigma_{2} \times p=\left(\left(t^{2}-1\right)(t y-z) x+21 z\right) .
$$

Now we have $\tilde{f} \equiv \bar{f}_{1} \times \bar{f}_{2} \bmod p^{2}$. This time the new error $e_{2}=\tilde{f}-\bar{f}_{1} \times \bar{f}_{2} \bmod \langle\tilde{m}\rangle$ is zero, so we have $\tilde{f}=\bar{f}_{1} \times \bar{f}_{2}$. To complete the factorization of $f$ we have $f=l c_{x, y}(f) \times$ $\operatorname{monic}\left(\bar{f}_{1}\right) \times \operatorname{monic}\left(\bar{f}_{2}\right)$, thus

$$
f=\left(t^{3}-t\right)\left(x y+20 z x-\frac{z}{t^{2}-1}\right)\left(x y-\frac{z x}{t}+\frac{21 z}{t^{3}-t}\right)
$$

and we are done.

\section{PROBLEMS}

In the example we mentioned that the evaluation point must satisfy certain conditions in order for the algorithm to work properly. Another issue is the defect of the algebraic function field $L$ which is the biggest denominator of any algebraic integer in $L$ (See $[1,9]$ ). Here we identify all problems.

\subsection{The Defect}

Unlike factorization over $\mathbb{Q}$, when factoring a polynomial $\tilde{f}$ over the algebraic field $L$, the leading coefficient of a factor $\tilde{f}_{i}$ in the variables $x_{1}, \ldots, x_{v}$ might not divide the leading coefficient of $\tilde{f}$, i.e. $\operatorname{lc}_{x_{1}, \ldots, x_{v}}\left(\tilde{f}_{i}\right) \nmid \mathrm{lc}_{x_{1}, \ldots, x_{v}}(\tilde{f})$ in $\mathbb{Z}\left[t_{1}, \ldots, t_{k}\right]$.
Example 4 Let $m=z^{2}-t^{3}, L=\mathbb{Q}(t)[z] /\langle m\rangle$ and $f=$ $x^{2}-t$. We have $\tilde{f}=f$ and

$$
f=\left(x-\frac{z}{t}\right)\left(x+\frac{z}{t}\right)=\frac{1}{t^{2}}(t x-z)(t x+z) .
$$

Here $\tilde{f}_{1}=t x-z$ but $l c_{x}\left(\tilde{f}_{1}\right)=t \nmid l c_{x}(\tilde{f})=1$.

The denominator $t$ in this example is a divisor of the defect of the algebraic function field $L$.

Theorem 1 (See [1]) The defect is the biggest square that divides $\Delta$, the discriminant of the algebraic field.

When $r=1$ (one field extension), $\Delta=\operatorname{res}_{z_{1}}\left(M, M^{\prime}\right.$ ) where $M=\tilde{m}_{1}$. For example, for $\tilde{m}=z^{2}-t^{3}$ we have $\Delta=\operatorname{res}_{z}\left(z^{2}-t^{3}, 2 z\right)=-4 t^{3}$ and hence $2 t$ is the defect.

Theorem 2 (See [1]) Let $d_{i}=\operatorname{deg}_{z_{i}}\left(m_{i}\right)$. The discriminant of $L$ is

$$
\Delta=\prod_{i=1}^{r-1} N_{1}\left(N_{2}\left(\ldots\left(N_{i-1}\left(\operatorname{discr}\left(\tilde{m}_{i}\right)^{d_{i+1} \ldots d_{r}}\right)\right) \ldots\right)\right)
$$

where $N_{i}(f)=\operatorname{res}_{z_{i}}\left(f, \tilde{m}_{i}\right)$ and $\operatorname{discr}\left(\tilde{m}_{i}\right)=\operatorname{res}_{z_{i}}\left(M_{i}, M_{i}^{\prime}\right)$ where $M_{i}=\tilde{m}_{i}$.

Suppose using Theorem 2 we have computed the discriminant $\Delta \in \mathbb{Z}\left[t_{1}, \ldots, t_{k}\right]$. Let $\delta \times D_{1}^{e_{1}} \times \cdots \times D_{k}^{e_{k}}$ be a squarefree factorization of $\Delta$ where $\delta \in \mathbb{Z}$. Since we want to avoid integer factorization, we choose $\mathbb{D}$ to be an integer multiple of the defect:

$$
\mathbb{D}=\delta \times D_{1}^{\left\lfloor\frac{e_{1}}{2}\right\rfloor} \times \cdots \times D_{k}^{\left\lfloor\frac{e_{k}}{2}\right\rfloor} .
$$

Theorem 3 (See [9]) If $\tilde{f}_{i}$ is a factor of $\tilde{f}$ and $\mathbb{D}$ is an integral multiple of the defect, then

$$
l c_{x_{1}, \ldots, x_{v}}\left(\tilde{f}_{i}\right) \mid \mathbb{D} \times l c_{x_{1}, \ldots, x_{v}}(\tilde{f})
$$

Remark 1 To compute an integral multiple of $\mathbb{D}$ in our algorithm, we compute $\Delta$ using Theorem 2 . We then do a square-free factorization of $\Delta / c$ where $c=\operatorname{cont}_{t_{1}, \ldots, t_{k}}(\Delta) \in$ $\mathbb{Z}$ is the integer content of $\Delta$, to find the biggest square $D$ which divides $\Delta / c$. We use $c \times D$ as the integral multiple of the defect.

Remark 2 As seen in Example 3, the leading coefficient of $\tilde{f}\left(\mathrm{lc}_{x_{1}, \ldots, x_{v}}(\tilde{f}) \in \mathbb{Z}\left[t_{1}, \ldots, t_{k}\right]\right)$ may not split among the leading coefficients of the factors. That is $\prod_{i=1}^{n} \operatorname{lc}_{x_{1}, \ldots, x_{v}}\left(\tilde{f}_{i}\right)$ may not divide $\mathbb{D}^{l} \times \mathrm{lc}_{x_{1}, \ldots, x_{v}}(\tilde{f})$ for any $l \in \mathbb{Z}^{+}$.

\subsection{Good and Lucky Evaluation Points}

\section{Definition 2 (Good Evaluation Points)}

Let $\alpha=\left(t_{1}=\alpha_{1}, \ldots, t_{k}=\alpha_{k}, x_{2}=\beta_{2}, \ldots, x_{v}=\beta_{v}\right) \in$ $\mathbb{Z}^{k+v-1}$ be the evaluation point that we choose in our algorithm to factor the univariate polynomial $\tilde{f}(\alpha)$. We impose the following conditions on $\alpha$. We say $\alpha$ is good if:

1. The leading coefficient of $\tilde{f}$ in the main variable $x_{1}$ and the leading coefficient of $\tilde{m}_{i}$ in $z_{i}$ do not vanish after evaluating at $\alpha$, i.e. $\operatorname{deg}_{x_{1}}(\tilde{f})=\operatorname{deg}_{x_{1}}(\tilde{f}(\alpha))$ and $\operatorname{deg}_{z_{i}}\left(\tilde{m}_{i}\right)=\operatorname{deg}_{z_{i}}\left(\tilde{m}_{i}(\alpha)\right)$.

2. $L(\alpha)$ remains a field so that we still have unique factorization of $\tilde{f}(\alpha)$. As an example, the evaluation point $t=1$ is not a good choice for our Example 2 because the minimal polynomial $z^{2}-t$ evaluated at this point is no longer irreducible. 
3. The polynomial $\tilde{f}$ evaluated at $\alpha$ remains square-free in $x_{1}$, i.e. $\operatorname{gcd}\left(\tilde{f}(\alpha), \tilde{f}^{\prime}(\alpha)\right)=1$ in $L(\alpha)\left[x_{1}\right]$, so that we can apply Hensel lifting.

4. The fourth condition on the evaluation point $\alpha$ is to be able to distribute factors of $l c_{x_{1}}(\tilde{f})$ to the monic univariate factors $u_{1}, \ldots, u_{n}$ where $u_{i} \in L(\alpha)\left[x_{1}\right]$ and

$$
\tilde{f}(\alpha)=l c_{x_{1}}(\tilde{f})(\alpha) \times u_{1} \times \cdots \times u_{n} .
$$

Suppose $\gamma \times \hat{l}_{1}^{e_{1}} \times \cdots \times \hat{l}_{m}^{e_{m}}$ is the factorization of $l c_{x_{1}}(\tilde{f})$ and $\mathbb{D}$ is the defect. Here $\gamma \in \mathbb{Z}\left[t_{1}, \ldots, t_{k}\right]$ and $\hat{l} \in$ $L\left[x_{2}, \ldots, x_{n}\right]$. Let $\beta=\mathbb{D} \times \gamma=\Omega \times \beta_{1}^{c_{1}} \times \beta_{2}^{c_{2}} \times \cdots \times$ $\beta_{k}^{c_{k}}$ where $\Omega \in \mathbb{Z}$ and $\beta \in \mathbb{Z}\left[t_{1}, \ldots, t_{k}\right]$. Let $\bar{d}_{i}=$ den $\left(1 / \hat{l}_{i}(\alpha)\right)$. In order to be able to uniquely distribute the factors of $\mathbb{D} \times l c_{x_{1}}(\tilde{f})$ to the univariate factors, we require that numbers in the set

$$
A=\left\{\beta_{1}(\alpha), \ldots, \beta_{k}(\alpha), \bar{d}_{1}, \ldots, \bar{d}_{m}\right\}
$$

have distinct prime divisors that do not divide $\Omega$ (See Example 5 below).

Similarly, a prime $p$ is said to be a good prime if conditions 1 and 3 above are satisfied modulo $p$.

Example 5 In Example 3 we have $l c_{x}\left(\tilde{u}_{1}\right)=19630325$, $l c_{x}\left(\tilde{u}_{2}\right)=22451$. We have $\beta_{1}=t-1, \beta_{2}=t+1, \hat{l}_{1}=$ ty $-z, \hat{l}_{2}=y+20 z$ and $\Omega=2$. We can not use the evaluation point $\alpha=(t=3, y=5)$ because the numbers in $A=\left\{2=(2), 4=(2)^{2}, 417=(3)(139), 9551=(9551)\right\} d o$ not have distinct prime divisors. Note that we can still distribute the last two factors of the leading coefficient using this evaluation point.

Remark 3 Condition 4 will not be satisfied, no matter what $\alpha$ is, if any two irreducible factors of $l_{x_{1}}(\tilde{f})$ have the same norm, i.e. $\exists i, j: \operatorname{norm}\left(\hat{l}_{i}\right)=\operatorname{norm}\left(\hat{l}_{j}\right)$ where $\hat{l}_{i}$ and $\hat{l}_{j}$ are irreducible factors of $\mathrm{lc}_{x_{1}}(\tilde{f})$. In this case, the denominators $\bar{d}_{i}=\operatorname{den}\left(1 / \hat{l}_{i}(\alpha)\right)$ and $\bar{d}_{j}=\operatorname{den}\left(1 / \hat{l}_{j}(\alpha)\right)$ will be images of the same polynomial norm $\left(\hat{l}_{i}\right)=\operatorname{norm}\left(\hat{l}_{j}\right)$ (See [6]). In this case we need to do something else. The simplest solution is to shift the variables $x_{2}, x_{3}, \ldots$ in the input polynomial by computing

$$
\tilde{f}:=\tilde{f}\left(x_{1}, x_{1}+c_{2} x_{2}, x_{1}+c_{3} x_{3}, \ldots, x_{1}+c_{v} x_{v}\right)
$$

for some $c_{i} \in \mathbb{Z}$. Now $\mathrm{lc}_{x_{1}}(\tilde{f}) \in \mathbb{Z}\left[t_{1}, \ldots, t_{k}\right]$, i.e. the leading coefficient of $\tilde{f}$ in $x_{1}$ will not involve any of the variables $x_{2}, x_{3}, \ldots, x_{v}$. The following is an example.

Example 6 Let $\tilde{m}=z^{2}-t$ and $\tilde{f}=((y+z) x+t)((y-z) x+$ $t)$. We have $l c_{x}(\tilde{f})=\hat{l}_{1} \times \hat{l}_{2}=(y-z)(y+z)$ and norm $\left(\hat{l}_{1}\right)=$ norm $\left(\hat{l}_{2}\right)=y^{2}-t$. If we choose $\alpha=(y=1, t=6)$ we will have $\bar{d}_{1}=\operatorname{den}\left(1 / \hat{l}_{1}(\alpha)\right)=5$ and $\bar{d}_{2}=\operatorname{den}\left(1 / \hat{l}_{2}(\alpha)\right)=5$ and the set $A=\{5,5\}$ will not have a set of distinct prime divisors. If we shift the variable $y$ to $x+3 y$, we will get $\tilde{f}:=\tilde{f}(x, x+3 y)=\left(x^{2}+(3 y+z) x+t\right)\left(x^{2}+(3 y-z) x+t\right)$ and $l c_{x}(\tilde{f})=1$.

Definition 3 (Lucky Evaluation Point) A good evaluation point $\alpha \in \mathbb{Z}^{v+k-1}$ is said to be lucky if it satisfies the following conditions, otherwise it is unlucky.

(i) The number of irreducible factors of $\tilde{f}(\alpha)$ over $L(\alpha)$ is the same as the number of irreducible factors of $\tilde{f}$. (ii) If $\hat{l}_{i} \mid l c_{x_{1}}\left(\tilde{f}_{j}\right)$ where $\tilde{f}_{j}$ is an irreducible factor of $\tilde{f}$ then $\operatorname{gcd}\left(\operatorname{den}\left(1 / \hat{l}_{i}(\alpha)\right), l c_{x_{1}}\left(\tilde{u}_{j}\right)\right) \neq 1$.

(iii) If $\beta_{i} \mid l c_{x_{1}}\left(\tilde{f}_{j}\right)$ then $\operatorname{gcd}\left(\beta_{i}(\alpha), l c_{x_{1}}\left(\tilde{u}_{j}\right)\right) \neq 1$

(iv) $\alpha$ does not annihilate any terms of any factor $\tilde{f}_{i}$ of $\tilde{f}$ (See Example 7 below).

Similarly, a good prime $p$ is said to be lucky if it does not annihilate any terms of any factor $\tilde{f}_{i}$ of $\tilde{f}$. If the evaluation point $\alpha$ or prime $p$ is unlucky, the algorithm must detect this and restart using a new good evaluation point.

Example 7 Let $\tilde{f}=\tilde{f}_{1} \times \tilde{f}_{2}$ where $\tilde{f}_{1}=x^{2}-(t-15) z x-$ $t z+1$ and $\tilde{f}_{2}=x^{3}-17 t z x+1$ where $z=\sqrt{t-1}$. Here the evaluation point $t=15$ is good but it is unlucky because it annihilates the term $(t-15) z x$ in $\tilde{f}_{1}$. Similarly, the prime $p=17$ is unlucky because the term $17 t z x$ in $\tilde{f}_{2}$ vanishes modulo $p$. Also, $t=0$ is unlucky because $\tilde{f}_{2}(t=0)$ factors.

Remark 4 Since we will use sparse interpolation to lift the integer coefficients of the factors computed using Hensel lifting, the evaluation point $\alpha$ and the prime $p$ must not $a n$ nihilate any terms in any factors of $\tilde{f}$. Unfortunately we will not be able to identify unlucky evaluation points and primes in advance. Instead, if $\alpha$ is unlucky or $p$ is unlucky and the form of any of the correcting polynomials $\sigma_{1}, \sigma_{2}, \ldots$ is wrong, the system of linear equations in the sparse interpolation would be inconsistent with high probability. To decrease the probability of choosing an evaluation point (or a prime) that annihilates terms in factors of $\tilde{f}$, one should choose $\alpha$ (and $p$ ) at random from a large set of evaluation points (or primes), e.g. $p=2^{31}-1$ and $\alpha \in \mathbb{Z}_{p}$ at random.

Remark 5 If $\alpha$ is unlucky and there are extraneous factors in the factorization of $\tilde{f}(\alpha)$ then Hensel lifting will fail with high probability. Hensel lifting may succeed modulo $p$ with low probability if the prime $p$ in Hensel lifting is also unlucky and results in extraneous factors in $\tilde{f} \bmod p$ corresponding to those of $\tilde{f}(\alpha)$.

Example 8 Suppose $\tilde{f}=x^{2}+17(t-1) z x-t^{2}$ and $z=$ $\sqrt{t+1}$. The evaluation point $\alpha=(t=1)$ is good but unlucky because $\tilde{f}$ is irreducible but $\tilde{f}(\alpha)=(x-1)(x+1)$. If we also chose $p=17$, Hensel lifting will succeed and return $(x-t)(x+t)$.

If Hensel lifting does not fail when $\alpha$ is unlucky, then we will not be able to lift the integer coefficients of factors of $\tilde{f}$ and the algorithm will restart by choosing a new evaluation point.

\subsection{Degree Bound for the Parameters}

In order to use Hensel lifting, we need to have bounds on the degrees of the parameters and variables in the factors of $\tilde{f}$. Unlike factorization over the rationals, $\operatorname{deg}_{t_{i}}\left(\tilde{f}_{i}\right)$ is not necessarily bounded by $\operatorname{deg}_{t_{i}}(\tilde{f})$.

Example 9 Let $m=z^{2}-\frac{1}{t^{3}}$ and $\tilde{f}=x^{2}-t$. We have

$$
\tilde{f}=\tilde{f}_{1} \tilde{f}_{2}=\left(x+t^{2} z\right)\left(x-t^{2} z\right) .
$$

Here $\operatorname{deg}_{t} \tilde{f}_{1}=\operatorname{deg}_{t} \tilde{f}_{2}=2>\operatorname{deg}_{t} \tilde{f}=1$. 
In [1], Abbott gives a possible bound $T_{i}$ on the degree of each factor in $t_{i}$ based on Trager's algorithm which is usually much bigger than the actual degrees of the factors. In our algorithm when we lift the parameter $t_{i}$ in the factorization of $\tilde{f}$, as soon as the factors have been lifted to the correct degree, the error would be zero with high probability and the algorithm succeeds. However if the evaluation point is unlucky, our algorithm will have to lift the parameter $t_{i}$ to the degree $T_{i}$ before realizing it. This happens with low probability. Instead of using the bad bound $T_{i}$, we start the algorithm with a heuristic bound $T$ for the degree of the parameters. Now Hensel lifting fails if either the evaluation point is unlucky or the heuristic bound $T$ is not big enough. In this case, we will double the heuristic bound, i.e. $T:=2 \times T$, and restart the algorithm by choosing a new evaluation point. In this way, we will eventually get a good evaluation point and a big enough bound $T$ and Hensel lifting will eventually succeed.

In our implementation we choose the initial bound $T$ based on the following conjecture from Abbott [1]:

$$
\operatorname{deg}_{t_{i}}\left(\tilde{f}_{i}\right) \leq \operatorname{deg}_{t_{i}}(\tilde{f})+\sum_{j=1}^{r} \operatorname{deg}_{t_{i}}\left(\tilde{m}_{j}\right) .
$$

\subsection{Numerical Bound}

Most algorithms that use Hensel lifting (See [10, 1]) either work over $\mathbb{Q}$ or work modulo a power of a prime which must be larger than twice the size of the largest integer coefficients in the factors of $\tilde{f}$. Abbott in [1] presents a bound for this but his bound is very poor. The following is an example from [1].

Example 10 Let $\tilde{m}=z^{2}-4 t-1$ and $\tilde{f}=x^{2}+x-t=$ $\left(x+\frac{1+z}{2}\right)\left(x+\frac{1-z}{2}\right)$. The bound given by Abbott for factoring $\tilde{f}$ is greater than 5000000 .

The poor bound leads to an unnecessarily large modulus which slows Hensel lifting down. Instead, we work modulo a machine prime $p$ and then lift the integer coefficients using our sparse $p$-adic lifting algorithm if necessary. We still need a bound for the case where $\alpha$ is unlucky and Hensel lifting has not detected this due to the unlucky choice of the prime $p$ (See Example 8). For this, we choose a heuristic bound $B$. Any good estimate for $B$ will work. Now if the sparse $p$-adic lifting fails, either $\alpha$ is unlucky or $p$ is unlucky or the bound $B$ is not big enough. In this case, we square the bound, i.e. $B:=B^{2}$, and restart the algorithm by using a new evaluation point $\alpha$ and new prime $p$. In this way, we will eventually get a lucky evaluation point and a lucky prime and a bound big enough to lift the integer coefficients.

\section{THE ALGORITHM}

\section{Algorithm efactor}

Input: $f \in L\left[x_{1}, x_{2}, \ldots, x_{v}\right]$ where $L$ is the algebraic function field.

Output: Factorization of $f: f=l \times f_{1}^{e_{1}} \times \cdots \times f_{n}^{e_{n}}$ where $f_{i}$ is a monic irreducible polynomial and $l=\mathrm{lc}_{x_{1}, \ldots, x_{v}}(f)$.

1: If $f \in L$, return $f$.

2: Let $c=\operatorname{cont}_{x_{1}}(f) \in L\left[x_{2}, \ldots, x_{n}\right]$ be the content of $f$. If $c \neq 1$ then factor $c$ and $f / c$ separately using Algorithm efactor and return the combined result.

3: Do a square-free factorization of $f$. Call algorithm 1 on each square-free factor and return the result.
Algorithm 1: Main algorithm

Input: Square-free $f \in L\left[x_{1}, x_{2}, \ldots, x_{v}\right]$ where $\operatorname{cont}_{x_{1}}(f)=1$.

Output: Factorization of $f: f=l \times f_{1} \times f_{2} \times \cdots \times f_{n}$ where $f_{i}$ is monic and $l=\mathrm{lc}_{x_{1}, \ldots, x_{v}}(f)$.

1: Compute $\tilde{f}$ (See Definition 1).

2: Compute $\mathbb{D}$, an integral multiple of the defect of the algebraic field $L$ (See Theorem 2).

3: if $v=1$ (univariate case) then

4: Call algorithm 3 on $\tilde{f}$ and $\mathbb{D}$ and return the result.

5: end if

6: Choose a heuristic bound $B$ for the largest integer coefficient in the factors of $\tilde{f}$.

7: Let $T=\max _{i=1}^{k}\left(\operatorname{deg}_{t_{i}}(\tilde{f})+\sum_{j=1}^{r} \operatorname{deg}_{t_{i}} \tilde{m}_{j}\right)$ (Heuristic bound on the degree of $\tilde{f}$ in any parameter: $A b$ bott's conjecture)

8: Factor $\mathrm{lc}_{x_{1}}(\tilde{f}) \in L\left[x_{2}, \ldots, x_{v}\right]$ by calling algorithm efactor. Let $\operatorname{lc}_{x_{1}}(\tilde{f})=\gamma \times l_{1}^{e_{1}} \times l_{2}^{e_{2}} \times \cdots \times l_{m}^{e_{m}}$ where $l_{i}$ is monic.

9: Compute $\tilde{l}_{i}$. Find $\bar{\gamma}, \bar{D} \in \mathbb{Z}\left[t_{1}, \ldots, t_{k}\right]$ s.t. $\bar{D} \times \operatorname{lc}_{x_{1}}(\tilde{f})=$ $\bar{\gamma} \times \prod_{i=1}^{m} \operatorname{lc}_{x_{2}, \ldots, x_{v}}\left(\tilde{l}_{i}\right)$. Update $\tilde{f}:=\bar{D} \times \tilde{f} .\left(\right.$ Note $\bar{D} \mid \mathbb{D}^{c}$ for some $c \in \mathbb{Z}^{+}$).

10: Main Loop: Choose a new good evaluation point $\alpha=\left(t_{1}=\alpha_{1}, t_{2}=\alpha_{2}, \ldots, t_{k}=\alpha_{k}, x_{2}=\beta_{2}, \ldots, x_{v}=\beta_{v}\right)$ that satisfies the requirements of Definition 2 in Section 3.2.

11: Let $D_{i}=\operatorname{den}\left(\tilde{l}_{i}(\alpha)^{-1}\right)$. If $\exists i, j: i \neq j, D_{i}=D_{j}$ then shift the variables $x_{2}, \ldots, x_{v}$ in $\tilde{f}$, call Algorithm 1 recursively on the shifted $\tilde{f}$, and undo the shift in the factors and return. (See Example 6).

12: Using Trager's algorithm factor $\tilde{f}(\alpha)$ to obtain $\tilde{f}(\alpha)=\Omega^{\prime} \times$ $u_{1} \times \cdots \times u_{n}$ where $\Omega^{\prime}=\operatorname{lc}_{x_{1}}(\tilde{f})(\alpha) \in \mathbb{Q}\left[z_{1}, \ldots, z_{r}\right]$. If $n=1$ then return $l \times \operatorname{monic}(\tilde{f}) \quad(\tilde{f}$ is irreducible $)$

13: Using algorithm 5 on inputs $\left\{u_{1}, \ldots, u_{n}\right\}, \operatorname{lc}_{x_{1}}(\tilde{f})=\bar{\gamma} \times \tilde{l}_{1}^{e_{1}} \times$ $\tilde{l}_{2}^{e_{2}} \times \cdots \times \tilde{l}_{m}^{e_{m}}$, the evaluation point $\alpha, \mathbb{D}$ and $\left\{D_{1}, \ldots, D_{m}\right\}$ compute the true leading coefficients of each univariate factor $\left\{\bar{l}_{1}, \bar{l}_{2}, \ldots, \bar{l}_{n}\right\}$. If this fails, go to step 10 . Note that $\tilde{f}$ may be updated in order to distribute the integer content of $\mathbb{D} \times$ $\operatorname{lc}_{x_{1}}(\tilde{f})$.

14: Compute $\delta, \hat{l} \in \mathbb{Z}\left[t_{1}, \ldots, t_{k}\right]$ s.t. $\delta \times \mathrm{lc}_{x_{1}, \ldots, x_{v}}(\tilde{f})=\hat{l} \times$ $\prod_{i=1}^{n} \operatorname{lc}_{x_{2}, \ldots, x_{v}}\left(\bar{l}_{i}\right) .\left(\delta \mid \mathbb{D}^{c}\right.$ for some $c \in \mathbb{Z}^{+}$and $\hat{l}$ is a factor of $l c_{x_{1}, \ldots, x_{v}}(\tilde{f})$ that is not in $\left.l_{1}, \ldots, l_{n}\right)$.

15: Set $\tilde{f}:=\delta \tilde{f}$. At this point we have

$$
\tilde{f}(\alpha)=\hat{l}(\alpha) \times\left(\bar{l}_{1}(\alpha) u_{1}\right) \times \cdots \times\left(\bar{l}_{n}(\alpha) u_{n}\right) .
$$

16: Choose a new good prime $p$ satisfying $\operatorname{lc}_{x_{1}}(\tilde{f}(\alpha)) \bmod p \neq 0$, $\operatorname{lc}_{z_{i}}\left(\tilde{m}_{i}(\alpha)\right) \bmod p \neq 0$ and $\tilde{f}(\alpha)$ is square-free modulo $p$.

17: Using algebraic Hensel lifting on inputs $\tilde{f}, \hat{l}$, the set of univariate images $\left\{u_{1}, \ldots, u_{n}\right\}$, the set of corresponding true leading coefficients $\left\{\bar{l}_{1}, \bar{l}_{2}, \ldots, \bar{l}_{n}\right\}$, the prime $p$, the bound $T$ and the evaluation point $\alpha$, lift the variables $x_{2}, x_{3}, \ldots, x_{v}$ and the parameters $t_{1}, \ldots, t_{k}$ to obtain $\tilde{f}=\hat{l} \times \bar{f}_{1} \times \bar{f}_{2} \times \cdots \times$ $\bar{f}_{n} \bmod \left\langle\tilde{m}_{1}, \ldots, \tilde{m}_{r}, p\right\rangle$.

18: If Hensel lifting fails then Set $T:=2 \times T$ and go to Step 10 .

19: Call algorithm 2 on inputs $\tilde{f}, \bar{f}_{1}, \bar{f}_{2}, \ldots, \bar{f}_{n}, \hat{l}$, the prime $p$, the bound $B$ and $\left\{l_{1}, l_{2}, \ldots, l_{n}\right\}$. If this fails, set $B:=B^{2}$ and go to step 10 otherwise let $f_{1}^{\prime}, f_{2}^{\prime}, \ldots, f_{n}^{\prime}$ be the output s.t. $\tilde{f}=\hat{l} \times f_{1}^{\prime} \times \cdots \times f_{n}^{\prime}$ over $L$.

20: return $\operatorname{lc}_{x_{1}, \ldots, x_{v}}(f) \times \operatorname{monic}\left(f_{1}^{\prime}\right) \times \cdots \times \operatorname{monic}\left(f_{n}^{\prime}\right)$

\section{Algorithm 2: Sparse $p$-adic lifting}

Input: $\tilde{f}, \tilde{f}_{1}, \ldots, \tilde{f}_{n} \in L\left[x_{1}, \ldots, x_{v}\right], \hat{l} \in \mathbb{Z}\left[t_{1}, \ldots, t_{k}\right]$ and $p$ s.t. $\tilde{f}-\hat{l} \times \tilde{f}_{1} \times \tilde{f}_{2} \times \cdots \times \tilde{f}_{n}=0 \bmod \left\langle\tilde{m}_{1}, \ldots, \tilde{m}_{r}, p\right\rangle . \quad$ The numerical bound $B$ and $\left\{l_{1}, \ldots, l_{n}\right\}$ the set of the leading coefficients of the factors.

Output: Either FAIL, if the evaluation point is unlucky or polynomials $h_{1}, h_{2}, \ldots, h_{n}$ s.t. $\tilde{f}=\hat{l} \times h_{1} \times \cdots \times h_{n}$ over $L$.

1: Let $h_{i}$ be $\tilde{f}_{i}$ with its leading coefficient replaced by $l_{i}$.

2: Let $e=\tilde{f}-\hat{l} \times h_{1} \times \cdots \times h_{n} \bmod \left\langle\tilde{m}_{1}, \ldots, \tilde{m}_{r}\right\rangle$. (Note that $\left.\operatorname{deg}_{x_{1}}(e)<\operatorname{deg}_{x_{1}}(\tilde{f})\right)$ 
3: Let $P=p$.

4: Suppose $\tilde{f}_{i}=\sum_{j=1}^{T_{i}} a_{i j} M_{i j}$ with $a_{i j} \in \mathbb{Z}_{p}$ and $M_{i j}$ monomials.

5: Let $\sigma_{i}=\sum_{j=1}^{T_{i}} A_{i j} M_{i j}$ where $A_{i j}$ is an unknown coefficient.

6: while $e \neq 0$ and $P<2 B$ do

7: $\quad e^{\prime}=e / P($ exact division $)$

8: $\quad$ Let $p_{z}=e^{\prime}-\hat{l} \times \sum_{i=1}^{n} \sigma_{i} \frac{\prod_{j=1}^{n} h_{j}}{h_{i}} \bmod \left\langle\tilde{m}_{1}, \ldots, \tilde{m}_{r}\right\rangle$.

9: $\quad$ Solve for $A_{i j}$ s by collecting and equating coefficients of $p_{z}$ in $x_{1}, \ldots, x_{v}, t_{1}, \ldots, t_{k}$ and $z_{1}, \ldots, z_{r}$ to zero modulo $P$.

10: If the system of linear equations is inconsistent then return FAIL. (Annihilated term in the form due to the choice of the modulus)

11: $\quad$ Update $h_{i}:=h_{i}+\sigma_{i} \times P$ for $1 \leq i \leq n$.

12: $\quad$ Set $P:=P^{2}$

13: $\quad$ Set $e=\tilde{f}-\hat{l} \times h_{1} \times \cdots \times h_{n} \bmod \left\langle\tilde{m}_{1}, \ldots, \tilde{m}_{r}\right\rangle$.

14: end while

15: If $e=0$ then return $h_{1}, h_{2}, \ldots, h_{n}$ else return FAIL.

\section{Algorithm 3: Univariate factorization}

Input: Square-free $f \in L\left[x_{1}\right]$ and $\mathbb{D}$ the defect of $L$.

Output: Unique factorization of $f=\operatorname{lc}_{x_{1}}(f) \times f_{1} \times f_{2} \times \cdots \times f_{n}$ over $L$ s.t. $f_{i}$ is monic in $x_{1}$.

1: Compute $\tilde{f}$ (See Definition 1) and Let $\bar{l}=\mathrm{lc}_{x_{1}}(\tilde{f})$.

2: Choose a heuristic bound $B$ on the integer coefficients of the factors of $\tilde{f}$.

3: Let $T=\max _{i=1}^{k}\left(\operatorname{deg}_{t_{i}}(\tilde{f})+\sum_{j=1}^{r} \operatorname{deg}_{t_{i}} \tilde{m}_{j}\right)$

(Heuristic bound on the degree of $\tilde{f}$ in any parameter: $A b$ bott's conjecture).

4: Factor $\gamma=\mathbb{D} \times \bar{l} \in \mathbb{Z}\left[t_{1}, \ldots, t_{k}\right]$ over $\mathbb{Z}$ to obtain $\gamma=\Omega \times$ $\beta_{1}^{c_{1}} \times \cdots \times \beta_{k^{\prime}}^{c_{k^{\prime}}}$.

5: Main Loop: Choose a new good evaluation point $\alpha=\left(t_{1}=\right.$ $\left.\alpha_{1}, \ldots, t_{k}=\alpha_{k}\right)$ that satisfies the requirements of definition 2.

6: Using Trager's algorithm, factor $h=\tilde{f}(\alpha)=\bar{l}(\alpha) \times h_{1} \times$ $h_{2} \times \cdots \times h_{n}$ over the algebraic number field. Note that $\operatorname{lc}_{x_{1}}\left(h_{i}\right)=1$.

7: Compute $\tilde{h}_{i}$ and let $\bar{d}_{i}=\operatorname{lc}_{x_{1}}\left(h_{i}\right) \in \mathbb{Z}$. Find the biggest $e_{i j}$ s.t. $\beta_{i}^{e_{i j}} \mid \bar{d}_{j}$. Let $l_{i}=\beta_{1}^{e_{1 i}} \times \cdots \times \beta_{k^{\prime}}^{e_{k^{\prime} i}}$. Distribute $\Omega \in \mathbb{Z}$ to $l_{i}$ 's and if needed, update $\tilde{f}$ and $\tilde{h}_{i}$. At this point we have $l_{i}=\operatorname{lc}_{x_{1}}\left(\tilde{f}_{i}\right)$.

8: Compute $\delta, \hat{l} \in \mathbb{Z}\left[t_{1}, \ldots, t_{k}\right]$ s.t. $\delta \times \bar{l}=\hat{l} \times \prod_{i=1}^{n} l_{i} . \quad\left(\delta \mid \mathbb{D}^{c}\right.$ for some $c \in \mathbb{Z}$ and $\hat{l}$ is a factor of $l c_{x_{1}}(\tilde{f})$ that is not in $\left.l_{1}, \ldots, l_{n}\right)$

9: Let $\hat{f}=\delta \tilde{f}\left(\hat{f}(\alpha)=\hat{l}(\alpha) \times \tilde{h}_{1} \times \tilde{h}_{2} \times \cdots \times \tilde{h}_{n}\right)$.

10: Choose a new good prime $p$ satisfying $\operatorname{lc}_{x_{1}}(\tilde{f}(\alpha)) \bmod p \neq 0$, $\operatorname{lc}_{z_{i}}\left(\tilde{m}_{i}(\alpha)\right) \bmod p \neq 0$ and $\tilde{f}(\alpha)$ is square-free modulo $p$.

11: Lift the parameters $\left\{t_{1}, \ldots, t_{k}\right\}$ in $\hat{f}(\alpha)-\hat{l} \times \tilde{h}_{1} \times \tilde{h}_{2} \times \cdots \times$ $\tilde{h}_{n} \equiv 0 \bmod p$ using Hensel lifting with $l_{i} \in \mathbb{Z}\left[t_{1}, \ldots, t_{k}\right]$ as the true leading coefficient of $\tilde{h}_{i}$ and $T$ as the degree bound. If this fails, set $T:=2 \times T$ and go to step 5 (unlucky evaluation point).

12: Call algorithm 2 on inputs $\hat{f}, \tilde{h}_{1}, \tilde{h}_{2}, \ldots, \tilde{h}_{n}, \hat{l}$, the prime $p$, $\left\{l_{1}, \ldots, l_{n}\right\}$ and $B$. If this fails, set $B:=B^{2}$ and go to step 5 (main loop) otherwise let $f_{1}^{\prime}, f_{2}^{\prime}, \ldots, f_{n}^{\prime}$ be the output s.t. $\hat{f}=$ $\hat{l} \times f_{1}^{\prime} \times \cdots \times f_{n}^{\prime}$ over $L$.

13: return $\operatorname{lc}_{x_{1}}(f) \times \operatorname{monic}\left(f_{1}^{\prime}\right) \times \cdots \times \operatorname{monic}\left(f_{n}^{\prime}\right)$.

\section{Algorithm 4: Distinct prime divisors}

\section{Input: A set $\left\{a_{1}, a_{2}, \ldots, a_{n}\right\}$ where $a_{i} \in \mathbb{Z}$.}

Output: Either FAIL or a set of divisors $\left\{d_{1}, d_{2}, \ldots, d_{n}\right\}$ s.t. $d_{i} \neq 1$ and $d_{i} \mid a_{i}$ and $\forall j \neq i: \operatorname{gcd}\left(d_{i}, d_{j}\right)=1$.

1: for $i$ from 1 to $n$ do

2: $\quad$ Let $d_{i}=a_{i}$.

3: $\quad$ for $j$ from 1 to $i-1$ do

4: $\quad$ Let $g=\operatorname{gcd}\left(d_{i}, d_{j}\right)$.

5: $\quad$ Set $d_{i}:=d_{i} / g$ and $d_{j}:=d_{j} / g$

6: $\quad$ Let $g_{1}=\operatorname{gcd}\left(g, d_{i}\right)$ and $g_{2}=\operatorname{gcd}\left(g, d_{j}\right)$. (Either $g_{1}=1$ or $g_{2}=1$ )

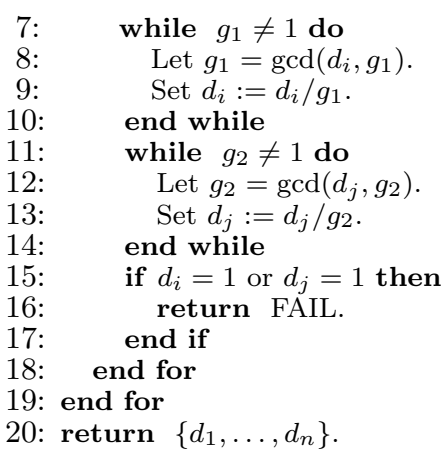

\section{Algorithm 5: Distributing leading coeffi- cients}

Input: $\tilde{f}$ and $U=\left\{u_{1}, u_{2}, \ldots, u_{n}\right\}$, the set of monic univariate factors where $u_{i} \in L(\alpha)\left[x_{1}\right] . \quad l=\gamma \times l_{1}^{e_{1}} \times l_{2}^{e_{2}} \times \cdots \times$ $l_{m}^{e_{m}}$ the non-monic factorization of $l=\operatorname{lc}_{x_{1}}(\tilde{f})$ where $\gamma \in$ $\mathbb{Z}\left[t_{1}, \ldots, t_{k}\right]$. The evaluation point $\alpha$ and $\mathbb{D}$ the defect of the algebraic field. $\left\{D_{1}, \ldots, D_{m}\right\}$ where $D_{i}=\operatorname{den}\left(l_{i}(\alpha)^{-1}\right)$.

Output: Either FAIL, if the leading coefficient is unlucky or $\left\{\hat{l}_{1}, \hat{l}_{2}, \ldots, \hat{l}_{n}\right\}$ where $\hat{l}_{i} \in L\left[x_{2}, \ldots, x_{v}\right]$ is the true leading coefficient of $u_{i}$ in $x_{1}$ together with possibly updated $\tilde{f}$.

1: Let $\beta=\mathbb{D} \times \gamma=\Omega \times \beta_{1}^{c_{1}} \times \beta_{2}^{c_{2}} \times \cdots \times \beta_{k^{\prime}}^{c_{k^{\prime}}}$ where $\Omega \in \mathbb{Z}$.

2: Let $d_{i}=\operatorname{den}\left(u_{i}\right)$ and $\mu_{i}=\beta_{i}(\alpha)$.

3: Let $\left\{p_{1}, \ldots, p_{m}, q_{1}, \ldots, q_{k^{\prime}}\right\}$ be the output of algorithm 4 on input $\left\{D_{1}, \ldots, D_{m}, \mu_{1}, \ldots, \mu_{k^{\prime}}\right\}$. If this fails, return FAIL.

4: For all $1 \leq i \leq m$, let $g_{i}=\operatorname{gcd}\left(\Omega, p_{i}\right)$ and Set $p_{i}:=p_{i} / g_{i}$. If $p_{i}=1$ then return FAIL.

5: For all $1 \leq i \leq k^{\prime}$, let $g_{i}^{\prime}=\operatorname{gcd}\left(\Omega, q_{i}\right)$ and Set $q_{i}:=q_{i} / g_{i}^{\prime}$. If $q_{i}=1$ then return FAIL.

6: for each $d_{j}$ do

7: $\quad$ for $i$ from 1 to $m$ do

8: $\quad$ Let $g_{1}=\operatorname{gcd}\left(d_{j}, p_{i}\right)$.

9: $\quad$ Set $e_{j i}^{\prime}=0$.

10: $\quad$ while $g_{1} \neq 1$ do

11: $\quad$ Set $e_{j i}^{\prime}:=e_{j i}^{\prime}+1$.

12: $\quad$ Set $d_{j}=d_{j} / g_{1}$.

13: $\quad$ Set $g_{1}=\operatorname{gcd}\left(d_{j}, g_{1}\right)$

14: $\quad$ end while

15: end for

16: $\quad$ for $i$ from 1 to $k^{\prime}$ do

17: $\quad$ Let $g_{2}=\operatorname{gcd}\left(d_{j}, q_{i}\right)$.

18: $\quad$ Set $c_{j i}^{\prime}=0$.

19: $\quad$ while $g_{2} \neq 1$ do

20: $\quad$ Set $c_{j i}^{\prime}:=c_{j i}^{\prime}+1$.

21: $\quad$ Set $d_{j}=d_{j} / g_{2}$.

22: $\quad$ Set $g_{2}=\operatorname{gcd}\left(d_{j}, g_{2}\right)$.

23: $\quad$ end while

24: end for

25: end for

26: for $i$ from 1 to $m$ do

27: $\quad$ if $\sum_{j=1}^{n} e_{j i}^{\prime} \neq e_{i}$ then return FAIL.

28: end for

29: Let $\hat{l}_{i}=\beta_{1}^{c_{i 1}} \beta_{2}^{c_{i 2}} \ldots \beta_{k^{\prime}}^{c_{i k^{\prime}}} l_{1}^{e_{i 1}} l_{2}^{e_{i 2}} \ldots l_{m}^{e_{i m}}$. Distribute $\Omega \in \mathbb{Z}$ to $\hat{l}_{i}$ s and if needed update $\tilde{f}$.

30: return $\left\{\hat{l}_{1}, \hat{l}_{2}, \ldots, \hat{l}_{n}\right\}$.

Remark 6 In our implementation of algorithm 1, we first choose an evaluation point and compute a univariate factorization then factor $\mathrm{lc}_{x_{1}}(\tilde{f})$. This is because if $\tilde{f}$ is irreducible, then we do not bother factoring the leading coefficient which might be a big polynomial.

\section{Description of Algorithm 2}

In algorithm 2, we have

$$
\tilde{f}-\hat{l} \times \tilde{f}_{1} \times \tilde{f}_{2} \times \cdots \times \tilde{f}_{n} \equiv 0 \bmod \left\langle p, \tilde{m}_{1}, \ldots, \tilde{m}_{r}\right\rangle .
$$


Note, if $\tilde{m}_{i}$ is not monic, the reduction modulo $\left\{\tilde{m}_{1}, \ldots, \tilde{m}_{r}\right\}$ does not introduce fractions in the parameters because of $\hat{l}$. Let $e_{1}=\hat{l} \times \tilde{f}-\tilde{f}_{1} \times \cdots \times \tilde{f}_{n} \bmod \left\langle\tilde{m}_{1}, \ldots, \tilde{m}_{r}\right\rangle$. We know that $p \mid e_{1}$. If $e_{1}=0$ then we are done. We want to find polynomials $\sigma_{1}, \ldots, \sigma_{n}$ s.t.

$$
\tilde{f}-\hat{l} \times\left(\tilde{f}_{1}+\sigma_{1} p\right)\left(\tilde{f}_{2}+\sigma_{2} p\right) \ldots\left(\tilde{f}_{n}+\sigma_{n} p\right) \equiv 0 \bmod p^{2} .
$$

Expanding the above expression and reducing modulo the set of minimal polynomials results in $g \equiv 0 \bmod p$ where

$$
g=\hat{l} \times\left(\sigma_{1} \tilde{f}_{2} \tilde{f}_{3} \ldots \tilde{f}_{n}+\cdots+\sigma_{n} \tilde{f}_{1} \tilde{f}_{2} \ldots \tilde{f}_{n-1}\right)-\frac{e}{p} .
$$

We assume that the terms in $\sigma_{i}$ are the same as the terms in $\tilde{f}_{i}$ with the integer coefficient replaced by an unknown. We compute the polynomial $g$ and equate each coefficient in $z_{1}, \ldots, z_{r}, t_{1}, \ldots, t_{k}, x_{1}, \ldots, x_{v}$ to zero. This gives us a linear system which has a unique solution because we already know the exact leading coefficient in the main variable of each factor $\tilde{f}_{i}$ and uniqueness is guaranteed by Hensel's lemma. After solving this system we will obtain the correction polynomials $\sigma_{1}, \ldots, \sigma_{n}$. We update each factor $\tilde{f}_{i}:=\tilde{f}_{i}+\sigma_{i} p$. Now we have

$$
\tilde{f}-\hat{l} \times \tilde{f}_{1} \times \tilde{f}_{2} \times \cdots \times \tilde{f}_{n} \equiv 0 \bmod p^{2} .
$$

We repeat this non-linear lifting algorithm until $p^{2^{k}}>2|B|$ where $B$ is the heuristic bound chosen in Algorithm 1 for the integer coefficients in the factors of $\tilde{f}$. Thus if there are no extraneous factors and no annihilated terms caused by the choice of primes and evaluation points, the algorithm will not depend on a bound on the size of the coefficients in the factor of $\tilde{f}$ which could be big.

Remark 7 In Step 8 of algorithm 3 and Step 14 of algorithm 1 , we compute $\hat{l} \in \mathbb{Z}\left[t_{1}, \ldots, t_{k}\right]$ which is the factor of the leading coefficient of $\tilde{f}$ in all the variables which does not show up in the leading coefficient of any factors of $\tilde{f}$.

Example 11 Let $m=z^{2}-\frac{1}{t}$ and $f=x^{2}-\frac{1}{t}$. We have $\tilde{m}=t z^{2}-1$ and $\tilde{f}=t x^{2}-1$. The factorization of $\tilde{f}$ is

$$
\tilde{f}=t(x-z)(x+z) \text {. }
$$

Here $l_{1}=l_{2}=1$ and $\hat{l}=t$. We have $\hat{l} \mid l c_{x}(\tilde{f})$ but $\hat{l} \nmid l_{i}$.

Remark 8 The bottleneck of Hensel lifting algorithm is solving the Diophantine equations. One can solve these Diophantine equations using sparse interpolation with a similar technique as in algorithm 2. Here is an example.

Example 12 Let $\check{m}=z^{2}-(t-1)^{3}$ and

$$
\tilde{f}=\left(t^{3}-t-t^{2}+1\right) x^{2}-x(2 t+1) z-t^{4}+t^{2} .
$$

Suppose we choose the evaluation point to be $t=4$. We compute the univariate factors using Trager's algorithm and after computing and attaching the leading coefficients of the factors we have

$$
\begin{aligned}
\hat{f} & =(t-1)^{2} \tilde{f}, \\
\tilde{f}_{1} & =\left(t^{3}-t-t^{2}+1\right) x+16 z, \\
\tilde{f}_{2} & =\left(t^{2}-2 t+1\right) x-5 z,
\end{aligned}
$$

where $\hat{f}-\tilde{f}_{1} \tilde{f}_{2} \equiv 0 \bmod (t-4)$. Now we start Hensel lifting. The first error polynomial is $e_{1}=\hat{f}-\tilde{f}_{1} \tilde{f}_{2}$. We have

$\frac{e_{1}}{t-4}=\left(3 t^{2} z-6 t z+3 z\right) x-t^{5}-2 t^{4}-8 t^{3}+46 t^{2}-55 t+20$.
Now we need to find two polynomials $\sigma_{1}$ and $\sigma_{2}$ s.t.

$$
\sigma_{2} \tilde{f}_{1}+\sigma_{1} \tilde{f}_{2}-\frac{e_{1}}{t-4} \equiv 0 \bmod (t-4) .
$$

Similar to algorithm 2, we can assume that $\sigma_{1}$ and $\sigma_{2}$ have the same monomials as $\tilde{f}_{1}$ and $\tilde{f}_{2}$ respectively and since we know that the leading coefficient of $\tilde{f}_{1}$ and $\tilde{f}_{2}$ are correct, the forms for $\sigma_{1}$ and $\sigma_{2}$ are $\sigma_{1}=A z$ and $\sigma_{2}=B z$. Using these forms and Equation 1 we construct and solve a linear system to obtain $A=8, B=-1$. We update $\tilde{f}_{1}:=\tilde{f}_{1}+\sigma_{1} \times(t-4)$ and $\tilde{f}_{2}:=\tilde{f}_{2}+\sigma_{2} \times(t-4)$ to get

$$
\begin{aligned}
& \tilde{f}_{1}=\left(t^{3}-t^{2}-t+1\right) x+16 z+8(t-4) z, \\
& \tilde{f}_{2}=\left(t^{2}-2 t+1\right) x-(t-4) z-5 z .
\end{aligned}
$$

This time the new error polynomial is $e_{2}=\hat{f}-\tilde{f}_{1} \tilde{f}_{2}$ and we have

$$
\frac{e_{2}}{(t-4)^{2}}=\left(t^{2} z-2 t z+z\right) x-t^{4}+2 t^{3}-2 t+1
$$

and

$$
\sigma_{2} \tilde{f}_{1}+\sigma_{1} \tilde{f}_{2}-\frac{e_{2}}{(t-4)^{2}} \equiv 0 \bmod (t-4)^{2} .
$$

The new assumed forms are

$$
\begin{aligned}
\sigma_{1} & =A z+B z(t-4), \\
\sigma_{2} & =C z t+D z .
\end{aligned}
$$

Again we construct a system of linear equations using Equation 2 and after solving this system we have $A=1, B=$ $0, C=0, D=0$. We update $\tilde{f}_{1}$ and $\tilde{f}_{2}$ and to obtain

$$
\begin{aligned}
& \tilde{f}_{1}=\left(t^{3}-t^{2}-t+1\right) x+z t^{2}, \\
& \tilde{f}_{2}=\left(t^{2}-2 t+1\right) x-z t-z .
\end{aligned}
$$

The new error polynomial $e_{3}=\hat{f}-\tilde{f}_{1} \tilde{f}_{2}$ is zero so $\tilde{f}=$ $l c_{x}(\tilde{f}) \times \operatorname{monic}\left(\tilde{f}_{1}\right) \times \operatorname{monic}\left(\tilde{f}_{2}\right)$ and we are done.

We do not use this method in our new algorithm for lifting parameters and variables. This is because it was always slower than solving the Diophantine equations using the traditional method. The reasons were:

1. The systems of linear equations in each step can be very big if the factors are dense.

Example 13 Suppose $\tilde{f}_{1}, \tilde{f}_{2}$ and $\tilde{f}_{1} \times \tilde{f}_{2}$ have $N_{1}, N_{2}$ and $N$ terms respectively. Then the system of linear equation has $N$ equations and as many as $N_{1}-1+$ $N_{2}-1$ unknowns.

2. As Hensel lifting progresses, usually, the error term gets smaller so solving the Diophantine equation is usually easier at the next step. But using sparse interpolation, as the Hensel lifting algorithm proceeds, each factor $\tilde{f}_{i}$ usually gets bigger because we add new terms, so the system of linear equations gets bigger which means Hensel lifting will be slower.

We do not have the second problem above for sparse interpolation in algorithm 2, when we lift integer coefficients, mainly because the forms of the $\sigma$ polynomials do not change due to the fact that only integer coefficients of factors of $\tilde{f}$ are being updated. 


\section{BENCHMARKS}

We have compared Maple 13 implementations of our new algorithm (efactor) with Maple's implementation of Trager's algorithm modified to use SparseModGcd (See [3]) for computing GCDs over $L$. This modified Maple implementation of Trager's algorithm is more efficient (See [5]).

The eight benchmark problems are available at http:// www.cecm.sfu.ca/ ${ }^{\sim}$ sjavadi/EFACT/benchmark.txt.

The timings are given in Table 1. All timings are in CPU seconds and were obtained on Maple 13 on a 64 bit AMD Opteron CPU @ $2.4 \mathrm{GHz}$, running Linux. In the table, $n$ is the number of variables, $r$ is the number of field extensions, $k$ is the number of parameters, $d$ is the total degree of $f, \# f$ is the number of terms in $f$ and \# $\tilde{f}$ is the number of terms in $\tilde{f}$. In all the problems, $f$ factors into two irreducible factors $f_{1}$ and $f_{2}$.

Problems 1 and 2 have large leading coefficients in the main variable $x$. Problems $3-5$ illustrate how Trager's algorithm is sensitive to the degree of the input and the number of variables. Problem 7 has many variables and parameters. Problem 8 has large integer coefficients. For problem 6, we multiplied the polynomial $f$ from Section 1 by one of its conjugates. Table 1 illustrates that Trager's algorithm did not finish in 50,000 seconds. In fact Maple had not computed the norm of the input polynomial after 50,000 seconds.

For each problem we used $p=3037000453$, a 31.5 bit prime, for Hensel lifting. For problems $3,4,5$ and $7, p$ is big enough so that there is no need to lift the integer coefficients using sparse $p$-adic lifting algorithm. For problems 1,2 and 6 , the number of lifting steps is one, i.e., $p^{2}>2\left\|\tilde{f}_{i}\right\|_{\infty}$. For the problem 8, the number of lifting steps is three, i.e. $p^{8}>2|| \tilde{f}_{i} \|_{\infty}$.

The last column in Table 1 is the time for computing

$$
\operatorname{gcd}\left(f_{1} f_{2}, f_{1}\left(f_{2}+1\right)\right)
$$

using our SparseModGcd algorithm in [3]. One can see that our factorization algorithm is often as fast as the GCD algorithm on a problem of comparable size, except for problem 6 . In problem 6 , almost all $(99 \%)$ of the time was factoring the univariate polynomial over $\mathbb{Q}(\sqrt{2}, \sqrt{3}, \sqrt{5}, \sqrt{7}, \sqrt{11})$ using Trager's algorithm.

\begin{tabular}{|c|c|c|c|c|}
\hline$\#$ & $(n, r, k, d, \# f, \# \tilde{f})$ & Trager & efactor & GCD \\
\hline 1 & $(2,2,1,17,191,6408)$ & 5500 & 259.91 & 47.47 \\
2 & $(2,2,1,22,228,12008)$ & 37800 & 296.74 & 56.90 \\
3 & $(2,2,2,10,34,34)$ & 120 & 0.22 & 0.16 \\
4 & $(2,2,2,12,34,34)$ & 571 & 0.31 & 0.19 \\
5 & $(3,2,2,10,69,69)$ & 5953 & 0.27 & 0.29 \\
6 & $(6,5,0,4,46,46)$ & $>50000$ & 88.43 & 1.93 \\
7 & $(5,2,1,10,15489,17052)$ & $>50000$ & 58.41 & 57.75 \\
8 & $(1,1,2,102,426,928)$ & 16427 & 72.10 & 7.71 \\
\hline
\end{tabular}

\section{Table 1: Timings (in CPU seconds)}

The percentages of timings for different parts of our new algorithm for these problems are presented in Table 2. In this table, the second column is the percentage of time spent on univariate factorization over $L(\alpha)$ using Trager's algorithm. The numbers in the third column correspond to the time spent on lifting variables and integer coefficients re-

\begin{tabular}{|c|c|c|c|}
\hline$\#$ & Univariate & Lifting & Sqr-free \\
\hline 1 & $0.30 \%$ & $(4.99 \%, 90.1 \%)$ & $4.01 \%$ \\
2 & $0.80 \%$ & $(7.82 \%, 84.42 \%)$ & $6.45 \%$ \\
3 & $51.61 \%$ & $(17.05 \%, 0 \%)$ & $19.35 \%$ \\
4 & $57.23 \%$ & $(22.03 \%, 0 \%)$ & $12.50 \%$ \\
5 & $42.86 \%$ & $(35.53 \%, 0 \%)$ & $19.41 \%$ \\
6 & $99.47 \%$ & $(0.31 \%, 0.52 \%)$ & $0.14 \%$ \\
7 & $0.80 \%$ & $(28,289 \%, 0 \%)$ & $67.41 \%$ \\
8 & $2.06 \%$ & $(91.68 \%, 5.47 \%)$ & $0.70 \%$ \\
\hline
\end{tabular}

Table 2: Timing (percentile) for different parts of efactor

spectively. And finally, numbers in the last column are the percentages of time spent on doing square-free factorizations of the inputs. One can see that the bottleneck of our new algorithm for the first two problems is the sparse $p$-adic lifting algorithm. This is because of the large number of terms in $\tilde{f}$.

\section{REFERENCES}

[1] J. A. Abbott. On the factorization of polynomials over algebraic fields. PhD thesis, School of Math. Sci., Univ. of Bath, England, 1989.

[2] Jürgen Gerhard and Ilias S. Kotsireas. Private communication.

[3] S. M. Mahdi Javadi and M. B. Monagan. A sparse modular gcd algorithm for polynomials over algebraic function fields. In Proceedings of ISSAC '07, pages 187-194. ACM, 2007.

[4] Erich Kaltofen. Sparse hensel lifting. In EUROCAL '85: European Conf. on Computer Algebra-Vol. 2, pages 4-17. Springer-Verlag, 1985.

[5] Michael Monagan. Computing polynomial greatest common divisors over algebraic number and function fields. http://www.cecm.sfu.ca/ pborwein/MITACS/ highlights/sparseGcd.pdf.

[6] Barry M. Trager. Algebraic factoring and rational function integration. In Proceedings of SYMSAC '76, pages 219-226. ACM, 1976.

[7] Paul S. Wang. Factoring multivariate polynomials over algebraic number fields. Mathematics of Computation, 30(134):324-336, 1976.

[8] Paul S. Wang. An improved multivariate polynomial factorization algorithm. Math. Comp., 32(144):1215-1231, 1978.

[9] P. J. Weinberger and L. P. Rothschild. Factoring polynomials over algebraic number fields. ACM Trans. Math. Softw., 2(4):335-350, 1976.

[10] Lihong Zhi. Algebraic factorization and gcd computation. Mathematics Mechanization and Applications, pages 325-342, 2000.

[11] Richard Zippel. Probabilistic algorithms for sparse polynomials. In Proceedings of EUROSAM 'r9, pages 216-226. Springer-Verlag, 1979. 